\title{
Ultrafast Emulation of Retinal Neuronal Circuits with Artificial VCSEL optical Neurons
}

\author{
Joshua Robertson ${ }^{1}$, Ewan Wade and Antonio Hurtado ${ }^{2}$ \\ Institute of Photonics, Dept. of Physics \\ University of Strathclyde \\ Technology and Innovation Centre \\ 99 George St., G1 1RD, Glasgow, UK \\ ${ }^{1}$ https://orcid.org/0000-0001-6316-5265 \\ ${ }^{2}$ https://orcid.org/0000-0002-4448-9034
}

systems and neuronal circuit simulators, here we have focused on the emulation of basic neural circuitry in the human retina. Spiking and non-spiking responses of different neurons, e.g. Photoreceptors, Bipolar Cells (BCs) and Retinal Ganglion Cells (RGCs) are reproduced optically using interconnected VCSELNeurons and at ultrafast speeds. This work therefore provides a first demonstration of key processing features of interest to neuromorphic computing platforms and highlight the diversity and versatility of VCSEL-Neurons for use in future neuroinspired processing networks.

\section{EXPERIMENTAL RESULTS}

Fig. 1(a) shows diagrams of the emulated retinal neuronal circuit and the non-spiking and spiking signals produced by the photoreceptors, BCs and the RGCs. BCs are non-spiking neurons connected to photoreceptors in the eye that fire different graded potentials dependent on the photoreceptor input. RGCs are spiking neurons synapsed to the BCs, that convert graded potentials into spike firing patterns that are transmitted to the brain (via the optic nerve). Two types of BCs and RGCs exist, creating circuits of opposite responses (ON and OFF-type). These yield different outputs under the presence or absence of incoming light stimuli [9-10]. Figs. 1(b) and 2 plot respectively the designed architecture and the setup developed in this work. These use three VCSEL-Neurons (M-, BC-, RGC-VCSELs) connected in series to emulate the different types of biological neurons forming the $\mathrm{ON}$ - and OFFtype BC+RGC retinal circuitry. Devices compatible with current optical communication networks operating at $1550 \mathrm{~nm}$ were used here with their wavelengths matched by tuning their operation temperature.

The first VCSEL (M-VCSEL) emulates the square potentials produced by photoreceptors in the eye in response to light stimuli. To do so, M-VCSEL is encoded with temporal perturbations (stimuli) of opposite signs, negative and positive for the ON- and OFF-type circuits, using a Mach-Zehnder amplitude modulator. The signals from M-VCSEL are launched into the second device, emulating a BC Neuron (BCVCSEL), injection-locking its output to M-VCSELs' light. In this situation, stimuli from M-VCSEL, trigger the firing of square-like signals (graded potentials) by BC-VCSEL. These will be of opposite sign (negative or positive) depending on the circuit emulated (ON- or OFF-type). BC-VCSEL's signals arrive into the third device (RGC-VCSEL), emulating an RGC Neuron, injection-locking it to BC-VCSEL. In the ON-type circuit, the graded potentials from BC-VCSEL trigger a short transition from injection-locking to unlocking regimes, and the RGC-VCSEL fires as a result a controlled spiking pattern. On 
the other hand, in the OFF-type circuit the positive graded potentials from BC-VCSEL cause a short transition from an unlocked (spiking) to an injection-locked (stable) regime producing a temporal window of spiking inhibition.
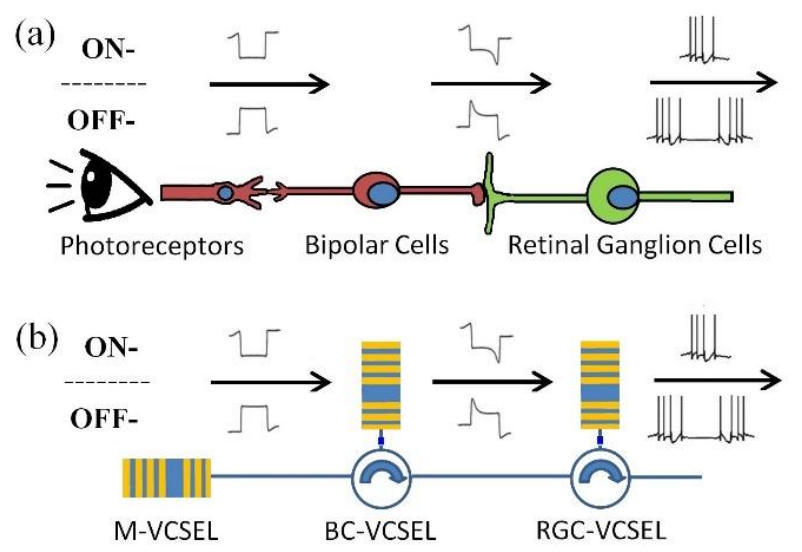

Fig. 1. (a) Schematic of the emulated retinal neuronal circuits formed by Photoreceptors, Bipolar Cells (BC) and Retinal Ganglion Cells (RGC). The signal conversion of the two different types of circuits is shown, ON- (top) and OFF- (bottom). (b) Diagram depicting the emulation of the neuronal circuits using three interconnected VCSEL-Neurons (M-/BC-/RGC-VCSELs).

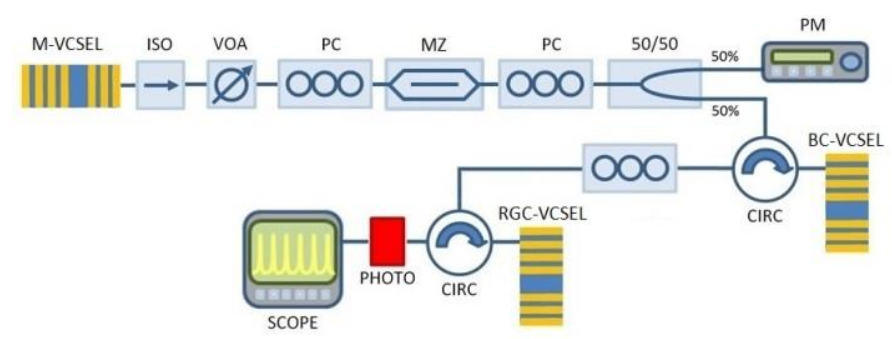

Fig. 2. Experimental Setup. M-, BC-, RGC-VCSEL: Master-, Bipolar Cell, Retinal Ganglion Cell-VCSEL, ISO: optical isolator, VOA: variable optical attenuator, PC: polarization controller, MZ: Mach-Zehnder amplitude modulator, PM: power meter, CIRC: optical circulator, PHOTO: photodetector, SCOPE: oscilloscope.

Figs. 3(a) and 3(b) respectively depict the outputs measured from each VCSEL-Neuron when operating in the ON- and OFF-type circuits. Fig. 3(a) shows that a 4.9ns-long (negative) stimuli encoded in M-VCSEL (Fig. 3(a,i), in black) triggers a (negative) graded potential at the output of BC-VCSEL (Fig. $3(\mathrm{a}, \mathrm{ii})$, in red) analogous to the non-spiking response of a $\mathrm{BC}$ Neuron. When the RGC-VCSEL receives the graded potential from BC-VCSEL, it reacts by firing spikes continuously until the graded potential is removed (Fig. 3(a,iii), in blue). Similarly, Fig. 3(b) plots the measured time-series from the 3 VCSEL-Neurons in the OFF-type architecture when 5.18nslong stimuli are encoded in M-VCSEL (Fig. 3(b,i), in black). The responses from $\mathrm{M}$ - and BC-VCSEL obtained here are opposite to those achieved in the ON-type circuit (Fig. 3(a)). The BC-VCSEL whose output (Fig. 3(b,ii), in red) produces a (positive) non-spiking graded potential triggers as well an opposite spiking response from RGC-VCSEL (Fig. 3(b,iii), in blue). The latter reacts producing a temporal window of spiking inhibition for the duration of the graded potentials from BC-
VCSEL. Importantly, the spiking responses produced at the output of both the ON- and OFF-type circuits were consistently produced across 60 consecutive stimulations as shown in the temporal maps of Figs. 3(a,iv) and 3(b,iv).
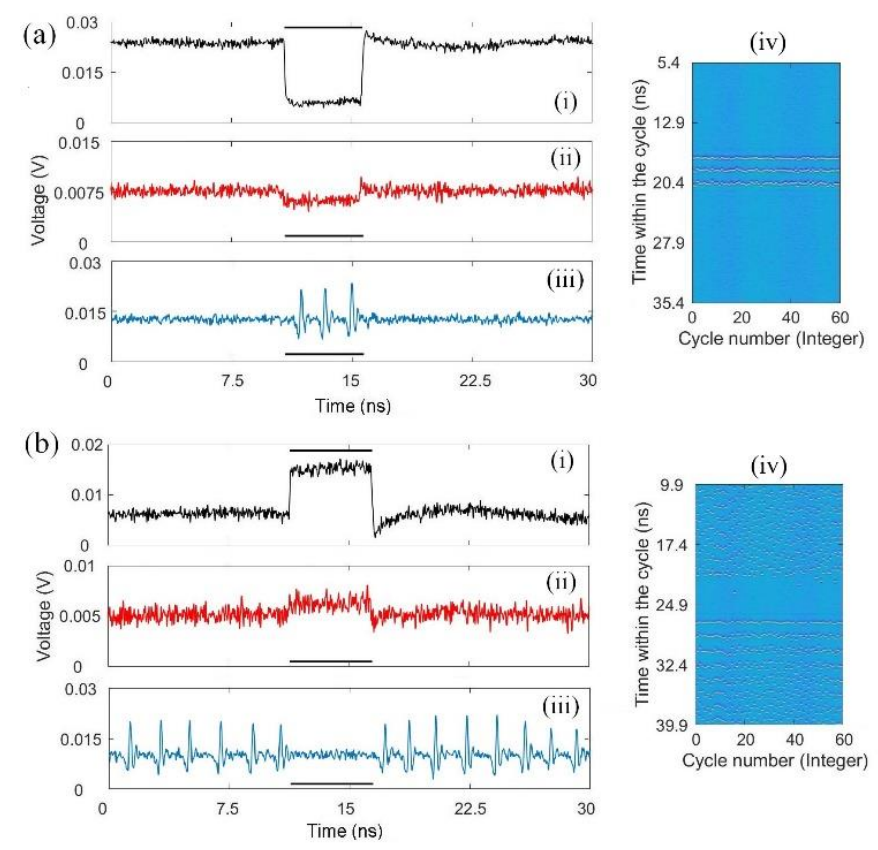

Fig. 3. Emulation of (a) ON- and (b) OFF-type retinal circuits with VCSELNeurons. Time-series from M-VCSEL ((i), black) BC-VCSEL ((ii), red) and RGC-VCSEL ((iii), blue) are plotted. The maps at the right hand side, plot the combined response at the output of the ON- (a,iv) and the OFF-type (b,iv) circuits under the arrival of 60 consecutive stimuli.

\section{CONCLUSION}

We emulate neural circuitry in the retina using a system of VCSEL-Neurons operating at the important telecom wavelength of $1550 \mathrm{~nm}$. We demonstrate that the biological signals from different types of neurons (BCs and RGCs) can be optically reproduced but at much faster (sub-ns/ns) timescales, $>7$ orders of magnitude faster than the millisecond response times of neurons. These results hold great promise for new compact, inexpensive neuromorphic photonic systems for future to braininspired computing platforms, neural circuit simulators and Artificial Intelligence systems.

\section{ACKNOWLEDGMENT}

The authors acknowledge support from the US Office of Naval Research Global (Grant ONRG-NICOP-N62909-18-1-2027), the European Commission (Grant 828841-ChipAI-H2020-FETOPEN-2018-2020) and the EPSRC Doctoral Training Partnership (EP/N509760).

\section{REFERENCES}

[1] P. R. Prucnal, et al., Neuromorphic Photonics, 1 ed., CRC Press, (2017).

[2] P. R. Prucnal, et al., Adv. Opt. Photon., vol. 8, pp. 228-299, (2016).

[3] M. J. Adams, et al., Chaos, vol. 20, 037102, (2010).

[4] A. Hurtado, et al., Appl. Phys. Lett., vol. 107, 241103, (2015).

[5] J. Robertson, et al., J. Sel. Top. Quantum. Electron., vol. 25, 5100307, (2019).

[6] J. Robertson, et al., Opt. Lett., vol. 42, 1560-1563, (2017).

[7] T. Deng et al., J. Sel. Top. Quantum. Electron., vol. 23, 1800408, (2017).

[8] T. Deng, et al., IEEE Access, vol. 6, pp. 67951-67958, Nov (2018).

[9] T. Euler, et al., Nat. Rev. Neuroscience, vol. 15, 507-519 (2014).

[10] R.H. Masland, Neuron, vol. 76, 266-280 (2012). 